\title{
Linear and non-linear plasma equilibrium responses on the JT-60U and TCV tokamaks
}

\author{
J.B. Lister ${ }^{\mathrm{a}, *}$, R. Khayrutdinov ${ }^{\mathrm{b}}$, D.J.N. Limebeer ${ }^{\mathrm{c}}$, V. Lukash ${ }^{\mathrm{d}}$, \\ Y. Nakamura ${ }^{\mathrm{e}}$, A. Sharma ${ }^{\mathrm{c}}$, F. Villone ${ }^{\mathrm{f}}$, J.P. Wainwright ${ }^{\mathrm{c}}$, R. Yoshino $^{\mathrm{e}}$ \\ ${ }^{a}$ Centre de Recherches en Physique des Plasmas, Association EURATOM-Confederation Suisse, EPFL, \\ 1015 Lausanne, Switzerland \\ ${ }^{\mathrm{b}}$ Triniti, Troitsk, Russian Federation \\ ' Imperial College of Science, Technology and Medicine, London, UK \\ ${ }^{\mathrm{d}}$ RRC Kurchatov Institute, Moscow, Russian Federation \\ e Naka Fusion Research Establishment, JAERI, Naka, Japan \\ ${ }^{\mathrm{f}}$ Association EURATOM/ENEA/CREATE, DAEIMI, University of Cassino, Cassino, Italy
}

\begin{abstract}
Although linear response models are useful for feedback controller design, their linear time-invariant properties cannot simulate the evolution of a full plasma discharge. A suitable code for this purpose is DINA, which has now been benchmarked against a complete set of experimental data from TCV control experiments in both the time and frequency domains. Experimental measurements of the plasma equilibrium dynamic response to poloidal field coil voltage variations have recently been performed on the JT-60U tokamak. These results have been compared with the RZIP rigid current displacement model, previously validated on TCV, but enhanced for the work described. (C) 2001 Elsevier Science B.V. All rights reserved.
\end{abstract}

Keywords: Plasma equilibrium; Tokamak; Simulation

\section{DINA simulations of TCV}

Considerable attention is being focused on the design of plasma position, current and shape controllers for the next generation tokamak-reactor designs, such as International Thermonuclear Experimental Reactor (ITER)-FEAT. The development of a new control algorithm for a new plasma

* Corresponding author. Tel.: + 41-21-693-3482; fax: + 4121-693-5176.

E-mail address: jo.lister@epfl.ch (J.B. Lister). configuration will require numerical analysis prior to experimental tests to optimise experimental time. It is, therefore, necessary to have a validated tokamak plasma simulator to test any new proposed plasma controller design. A first requirement of such a tool is its ability to model a real experimental plasma evolution with sufficient accuracy. Considerable success had been obtained in modelling the stationary phase of TCV discharges using two linear models, RZIP and CREATE-L [1-3]. However, to simulate a full discharge, we require a non-linear self-consistent model. DINA 
[4] is a tokamak plasma simulation code comprising a $1.5 \mathrm{D}$ axi-symmetric, time-dependent, resistive MHD and transport-modelling free boundary equilibrium solver in an externally imposed magnetic field and is a suitable candidate for such work. In this section, the validation of the DINA code against an extensive set of TCV plasma equilibrium response experiments is presented.

Initial test simulations using the DINA code indicated that two technical improvements were useful. Firstly, the feedback controller for these discharges has a high low-frequency gain to reduce any static offset. The feedback controller had to be initialised suitably at the start of the simulation, to start with a non-zero voltage on the integral term of the feedback controller, in order to avoid a transient, which could even drive the simulation unstable and end it prematurely, illustrated in Fig. 1. Secondly, it is very difficult to adjust the poloidal flux consumption between an experiment and a simulation, since the latter did not model the transport in most of the cases. Modelling the transport would not have helped matching the data, since a long adjustment of the transport coefficients by trial and error would have been necessary to obtain even modest agreement. A pragmatic solution was therefore adopted, feeding back the toroidal resistance to agree with the experiment. This adjustment did not perturb the investigation into the dynamic equilibrium response, which in most cases was assessed with the lowest frequency response (drift and offset) removed from both simulation and experimental data.

With these two improvements, 27 different discharges were simulated, with no adjustment of the DINA code, except for one minor numerical problem encountered for a particular discharge. Both limited and diverted plasmas were well modelled during the plasma current flattop for the rejection of external square-wave PF coil voltage pulses. Examples of the comparison between TCV and DINA responses are shown in Fig. 2. The agreement between the simulation and the experimental results was almost always within the noise width of the experimental data.

A complete single null diverted plasma discharge was also successfully simulated. The most significant difference between experiment and simulation was the penetration time of the poloidal flux, leading to a delayed onset of sawtoothing in the DINA simulation than in the experiment, Fig. 3. Since the plasma loop resistance has been adjusted to the experimental data, this implies that the initial penetration of the poloidal flux does not follow the toroidal resistivity and that care should be taken in using this assumption, when investigating the plasma current ramp-up phase. Different temperature profiles were used and the transport was enabled to confirm that this effect was not an artefact. The sawtooth frequency itself did vary according to the assumptions on the profiles, Fig. 3.

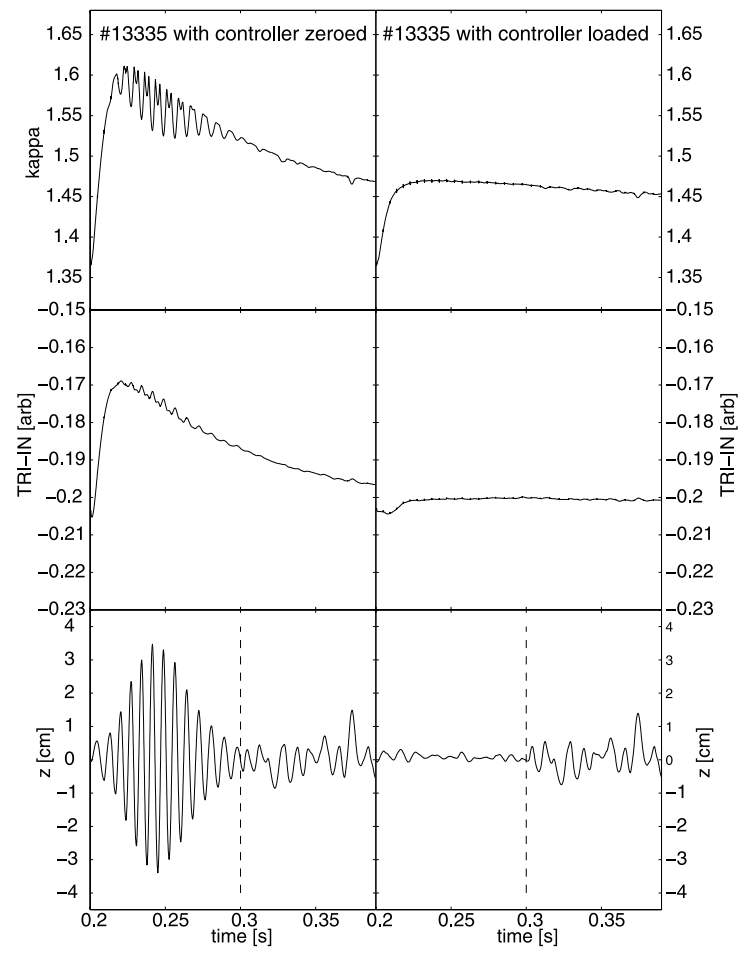

Fig. 1. Improvement to the controller initialisation caused a significant reduction to the transients at the start of the DINA simulation (right). Uninitialised (left), the transient included an increase to the vertical field decay index and hence to the elongation (top trace), resulting in an anomalous vertical instability showing up on the vertical movement trace (bottom), which caused a failure of the simulation in early tests. The middle trace shows the excursion of the control parameter for elongation. The start of the stimulation is indicated as a vertical dotted line. 


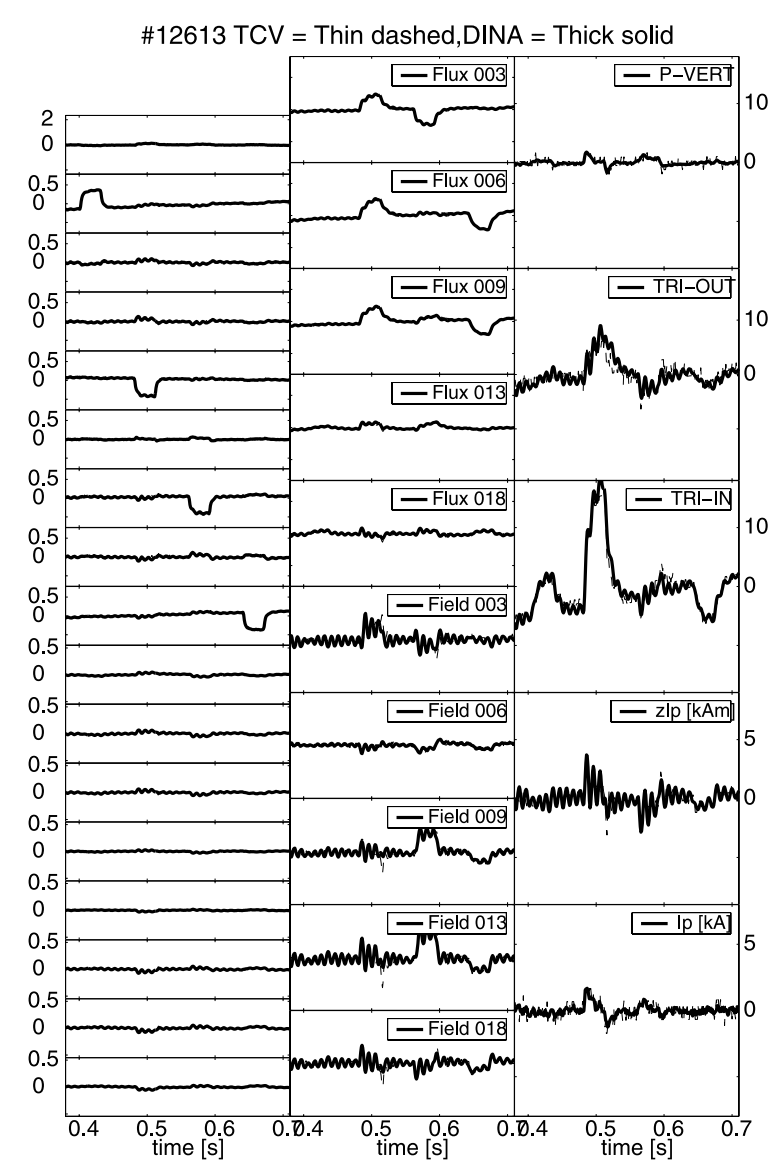

Fig. 2. Simulation of a diverted off-centred plasma, using separate E1, E4, E6, E8 coil stimulations in a single pulse. The signals are shown detrended: (left column) all 18 external PF coil currents, OH1, OH2, E1-E8, F1-F8; (middle column) flux loops \#3,6,9,13,18, magnetic probes \#3,6, 9, 13, 18; (right column) the five feedback control parameters.

Square pulse voltage stimulation of the $\mathrm{OH} 1$ and $\mathrm{OH} 2$ coils led to oscillations during the resulting experimental shape excursions, shown in Fig. 4 for the $\mathrm{OH} 1$ response. These had been identified previously as not reproducible by linear simulations [2], but are now well reproduced by the DINA simulation. The envelope increases around $400 \mathrm{~ms}$, showing that the TCV closed loop has gone unstable, with a growth time of 200-400 $\mathrm{ms}$. After $100 \mathrm{~ms}$, the oscillations become damped, when the closed loop is stable again. This change in closed loop stability is attributable to the increased vertical field decay index, which

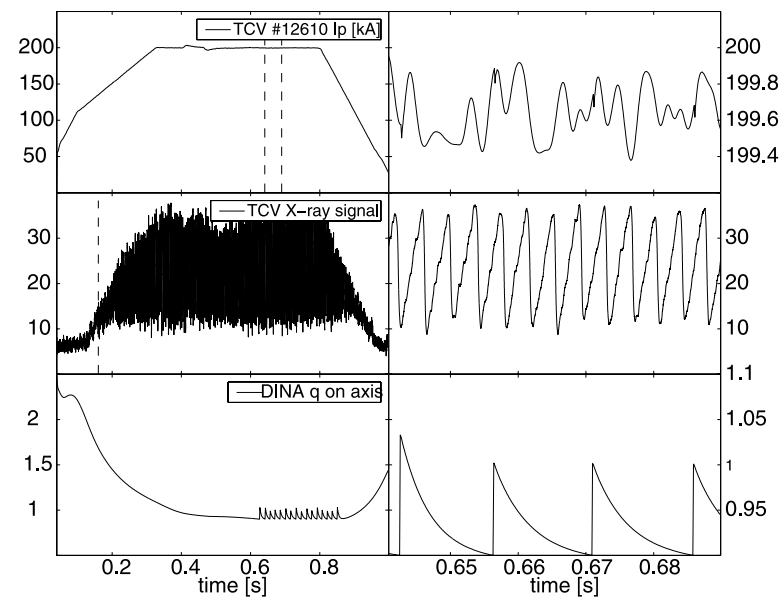

Fig. 3. Comparison between DINA simulation and TCV for the evolution of the sawtooth activity: left, the full pulse showing, from top to bottom: TCV Ip, TCV soft X-ray signal, DINA $q(0)$; right, an expanded view of the same signals.

causes an excursion of the plasma elongation. The increase of elongation from 1.45 to 1.53 occurs at the same time that the closed loop is unstable. This observation is an example of non-time-invariant behaviour of the experiment and the simulation, which can never be simulated by linearised models, for which time invariance is assumed.

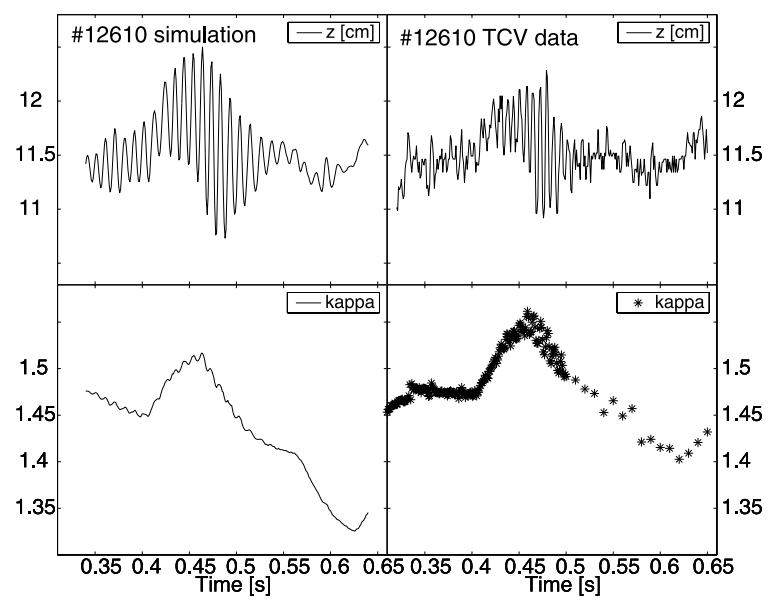

Fig. 4. Comparison between TCV (left) and the DINA simulation (right) for a large excursion due to a square voltage pulse $\mathrm{OH} 1$ stimulation. The vertical position goes closed loop unstable and returns to closed loop stable once the elongation is reduced. 
The complete set of 18 frequency stimulation experiments used to measure the open loop TCV plasma equilibrium response was also simulated in DINA and the results were analysed in an identical fashion to the experimental data [3]. In this more recent analysis, we used the coil voltages rather than the PF power supply demand voltages as the input signal, since this change actually renders the analysis more sensitive to differences between models, although being more noise prone. The frequency response of the DINA simulations agreed with the experimental results. The CREATE-L and RZIP models gave reasonable agreement as well, with the former showing fewer departures from the experimental results than the latter.

\section{JT-60U experiments}

The method [3] used on TCV to measure the open loop plasma equilibrium response during the closed loop control of the vertically unstable plasmas has been repeated on JT-60U. The locally linearised RZIP model of JT-60U has been rederived using a clean Lagrangian approach. This removed a previous asymmetry in the determining equations concerning the radial derivative of the plasma resistance and the coupling between major radius and plasma current, but left most terms unchanged, see Eq. 1 with a similar structure as [3].
A series of experiments was then performed with no plasma, in an Ohmic plasma and an NBIheated plasma, using multi-sine (five frequencies) excitation of the five $\mathrm{PF}$ coil voltages in the range 4-80 Hz, to determine the dynamic response of all diagnostics. The plasma-less model was fine-tuned to compensate for external circuitry and any constructional differences, to a precision of a few percent, beyond simple measurement accuracy. Different cost functions were explored to define a best tuning. This procedure was necessary to avoid biasing the measurements with plasma and attributing any differences found to plasma model errors. The open loop plasma response agreed well with the RZIP model, except for the plasma current response. Varying the plasma resistance derivative in the model showed that this small term is not experimentally determined by these experiments. Calibrating the values of the plasma inductance and plasma resistance in the model to agree with the experimental data showed that the effective resistance is much higher than the loop resistance and that the effective inductance is slightly lower than the nominal low frequency inductance. It is assumed that these observations can be explained by a skin effect. The resulting agreement between the experimental and modelled dynamic responses is shown in the Bode diagrams in Fig. 5 for the most important responses for designing a controller, namely the control parameters. The plasma-less response is shown as crosses for the

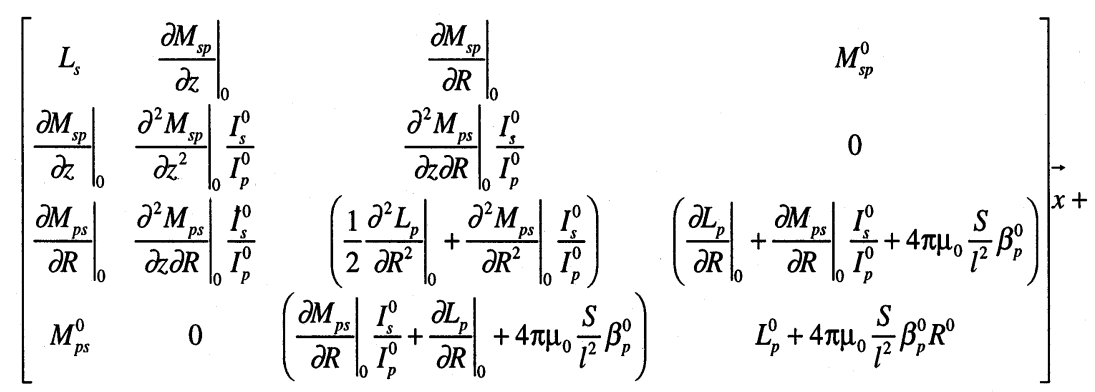

$$
\left[\begin{array}{cccc}
\Omega_{s} & 0 & 0 & 0 \\
0 & 0 & 0 & 0 \\
0 & 0 & 0 & \left.\frac{\partial \Omega_{p}}{\partial R}\right|_{0} \\
0 & 0 & \left.\frac{\partial \Omega_{p}}{\partial R}\right|_{0} & \Omega_{p}^{0}
\end{array}\right] \vec{x}=\left(\begin{array}{c}
\delta V_{s} \\
0 \\
-2 \pi \mu_{0} \frac{S}{l^{2}} I_{p}^{0} \beta_{p} \\
-4 \pi \mu_{0} \frac{S}{l^{2}} I_{p}^{0} R^{0} \beta_{p}
\end{array}\right) \quad \text { Eq (1) }
$$




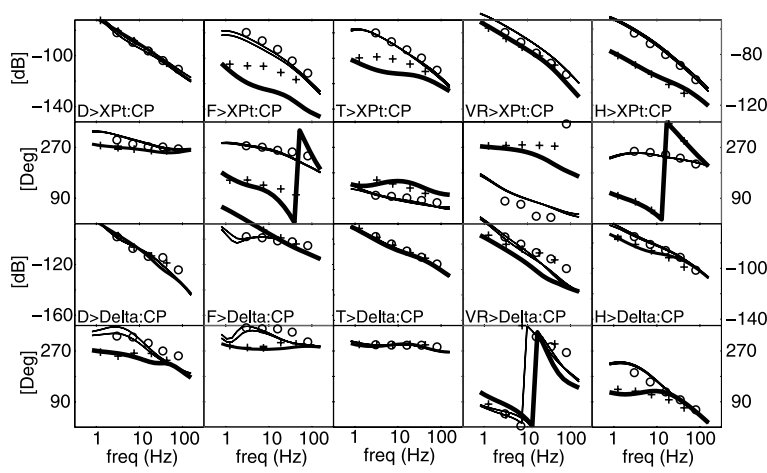

Fig. 5. Selected open loop responses from the JT-60U tokamak. The heavy lines and $(x)$ are the plasma-less model and data. The thin lines and $(\bigcirc)$ are the plasma data.

data and thick lines for the model. The plasma responses are indicated as circles for the data and thinner lines for the RZIP model.

Finally, the closed loop operation of JT-60U has been successfully simulated using the RZIP model and a linearised approximation of the JT$60 \mathrm{U}$ feedback controller. Empirical fine-tuning of this controller has been started to reduce some of the residual cross-couplings seen in the data.

\section{Acknowledgements}

Thanks go to the TCV and JT-60U teams without whom the experimental results would not be comparable with the models. Two of the authors (RK, VL) thank the CRPP for their hospitality during visits to TCV. Two of the authors (JBL, AS) thank JAERI-Naka for their hospitality during visits to JT-60U. This work was partly supported by the Swiss National Science Foundation and three of the authors (DJNL, AS, JPW) were partly supported by the United Kingdom EPSRC.

\section{References}

[1] F. Villone, P. Vyas, J.B. Lister, R. Albanese, Nucl. Fusion 37 (1997) 1395.

[2] P. Vyas, F. Villone, J.B. Lister, R. Albanese, Nucl. Fusion 38 (1998) 1043.

[3] A. Coutlis, et al., Nucl. Fusion 39 (1999) 663.

[4] R.R. Khayrutdinov, V.E. Lukash, J. Comp. Phy. 109 (1993) 193. 